\title{
A NOVEL CHARCOAL-INDUCED MODEL OF OBLITERATIVE BRONCHIOLITIS-LIKE LESIONS: IMPLICATIONS OF CHRONIC NONSPECIFIC AIRWAY INFLAMMATION IN THE DEVELOPMENT OF POSTTRANSPLANTATION OBLITERATIVE BRONCHIOLITIS
}

\author{
Allan G. L. Lee, MSc ${ }^{\mathrm{a}}$ \\ Florian M. Wagner, MD \\ Moy-Fong Chen, FRCPath ${ }^{\mathrm{b}}$ \\ Cyril Serrick, MSc \\ Adel Giaid, $\mathrm{PhD}^{\mathrm{b}}$ \\ Hani Shennib, MD
}

\begin{abstract}
Objectives: In this study, we describe the development of a nonallogeneic animal model of obliterative bronchiolitis-like lesions. Furthermore, we examined whether chronic rejection alone can lead to the development of obliterative bronchiolitis or whether additional nonspecific airway inflammation is required. Methods: Part I: Rats were intratracheally injected with $0.2 \mathrm{ml}$ of activated charcoal or sorbitol solution (carrier for charcoal control). Animals were put to death beginning at 2 weeks up to 20 weeks. Part II: Animals were divided into three groups: group I, underimmunosuppressed Brown Norway to Lewis lung allografts; group II, charcoal-treated underimmunosuppressed allografts; and group III, charcoal-treated rats. Animals were put to death at 3 months after transplantation. Results: Part I: In charcoal-laden bronchioles, subacute nonspecific airway inflammation was detected at 2 weeks. Slow, subclinical fibroproliferation ensued during the following weeks. Obliterative bronchiolitis-like lesions were observed in $80 \%$ of charcoal-treated animals at 12 weeks. Part II: Allografts developed extensive vascular lesions consistent with acute and chronic vascular rejection. Obliterative bronchiolitis-like lesions were scarcely detected. Charcoal-treated allografts demonstrated evidence of diffuse and severe obliterative bronchiolitis-like lesions. Conclusions: Transtracheal injection of activated charcoal into native lungs results in slowly progressive airway injury and inflammation leading to obliterative airway lesions. Inadequate immunosuppression primarily results in chronic vascular rejection but not obliterative bronchiolitis. Underimmunosuppressed allografts subjected to nonspecific airway inflammation develop obliterative airway lesions that are more prominent than in native lungs. This suggests that a cofactor to chronic rejection is likely necessary for the development of lung transplant obliterative bronchiolitis. (J Thorac Cardiovasc Surg 1998;115:822-7)
\end{abstract}

L ung transplantation has evolved into an accepted therapeutic option for patients with end-stage pulmonary diseases. However, development of

From The Montreal Lung Transplant Program, ${ }^{\mathrm{a}}$ and the Department of Pathology, McGill University, ${ }^{\mathrm{b}}$ Montreal, Quebec, Canada.

This work was funded in part by the Canadian Cystic Fibrosis Foundation, the Quebec Heart and Stroke Foundation, and the Quebec Lung Association. Allan G. L. Lee is a recipient of the Canadian Cystic Fibrosis Foundation. Hani Shennib is a senior scholar of the Fonds du Récherche en Santé Québec.

Received for publication Feb. 7, 1997; revisions requested May 21, 1997; revisions received Sept. 17, 1997; accepted for publication Sept. 17, 1997.

Address for reprints: Hani Shennib, MD, The Montreal General Hospital 1650 Cedar Ave., Suite L9-120, Montreal, Quebec H3G 1A4, Canada.

Copyright (c) 1998 by Mosby, Inc.

$0022-5223 / 98 \$ 5.00+0 \quad \mathbf{1 2 / 1 / 8 6 1 9 7}$ obliterative bronchiolitis (OB) has emerged as the most important factor limiting long-term survival after lung transplantation. ${ }^{1}$

Clinical and experimental findings suggest that the cause of lung transplant $\mathrm{OB}$ is multifactorial. Chronic rejection is considered to be the most important allogeneic stimulus. ${ }^{1}$ Nonallogeneic stimuli such as infections, lymphatic disruption, ischemia-reperfusion injury, and the side effects of immunosuppression may also play a role in the development of $\mathrm{OB} .^{1-5}$ Clinical investigation of these nonallogeneic factors is often hampered by the presentation of multiple complications, limited quantities of biopsied lung tissue, and small numbers of affected individuals for study. For these reasons and to examine the contribution of each of the possible contributing factors separately, it becomes imperative to develop reliable and reproducible experimental animal models of $\mathrm{OB}$. 
In this study we describe the development of a nonallogeneic animal model of OB-like lesions by instillation of a nonspecific airway irritant into the lungs of rats. Activated charcoal was used as the nonallogeneic stimulus. In addition, we examined whether chronic rejection alone or in combination with a nonallogeneic airway irritant is essential for extensive OB development in allografts.

\section{Material and methods}

Experimental design. Airway injury after intratracheal administration of activated charcoal was investigated in native (part I) and allografted (part II) lungs. In part I, we first determined the optimal dose of activated charcoal required to elicit mild progressive airway injury while limiting the number of deaths among the animals. Four groups of LEW rats ( $n=5$ per group) were anesthetized with an intraperitoneal injection of sodium pentobarbital (30 mg/kg), intubated under transluminescence with 16gauge intravenous catheters, and injected with a range of doses of Charcadote TFS-50 $(200 \mathrm{mg} / \mathrm{ml}$ activated charcoal per $200 \mathrm{mg} / \mathrm{ml}$ sorbitol, Pharmascierice Inc., Montreal) from $0.1 \mathrm{ml}$ up to $0.4 \mathrm{ml}$ consecutively. Animals experiencing respiratory difficulty were mechanically ventilated (Harvard Rodent Ventilator, Harvard Apparatus Co., Inc., S. Natick, Mass.). Animals were killed 4 weeks after charcoal administration. Once the optimal dose was determined, we then proceeded to characterize in detail the histopathologic evolution of airway lesions. LEW rats were divided into two groups: control $(n=25)$ and treatment $(n=25)$. Control animals were intratracheally injected with $0.2 \mathrm{ml}$ of sorbitol solution $(200 \mathrm{mg} / \mathrm{ml}$, carrier for charcoal). Treated animals were anesthetized, intubated, and intratracheally injected with $0.2 \mathrm{ml}$ of activated charcoal. Animals were sacrificed $(n=5)$ at 2, 4, 12,16 , and 20 weeks after sorbitol/charcoal administration.

In part II of the study, we analyzed whether chronically rejecting lung allografts are more susceptible to development of obliterative airway lesions after exposure to a nonspecific airway irritant. LEW rats were divided into three groups: group I, rats receiving allogeneic BN lung transplants $(n=5)$; group II, rats receiving allogeneic BN lung transplants followed by intratracheal administration of $0.2 \mathrm{ml}$ of activated charcoal 1 month after transplantation; and group III (control), LEW rats intratracheally injected with $0.2 \mathrm{ml}$ of activated charcoal $(n=5)$. All animals were killed at 3 months.

Young adult, male, specific pathogen-free (SPF) LEW (RT1) and BN (RT1n) rats weighing 250 to 300 gm were obtained from Harlan Sprague Dawley, Indianapolis, Indiana. Animals that survived the procedure were subsequently housed in isolation and SPF conditions in the animal care facilities of the Montreal General Hospital. Diets consisted of sterile rat chow and water. All animals were given humane care in compliance with the Animal Care Committee regulations of The Montreal General Hospital and McGill University and in compliance with the "Principles of Laboratory Animal Care" formulated by the National Society for Medical Research and the
"Guide for the Care and Use of Laboratory Animals" prepared by the National Academy of Sciences and published by the National Institutes of Health (NIH Publication No 85-23, revised 1985).

Lung transplantation and immunosuppression. Left lung allografts were orthotopically transplanted in the thorax according to the improved nonsuture external cuff technique described by our laboratory. ${ }^{6}$ Postoperative immunosuppression consisted of two single doses of cyclosporine A ( $25 \mathrm{mg} / \mathrm{kg} \mathrm{IM}$ ) given on days 2 and 3 only.

Histology. Resected left lungs were inflated and fixed in $4 \%$ paraformaldehyde for 6 hours, then switched to $15 \%$ phosphate buffered saline sucrose overnight and embedded in paraffin. Multiple-step sections from each paraffin block were cut and stained with hematoxylin and eosin, van Gieson's elastin, and Masson's trichrome stains. Multiple-step sections from different lung regions (hilum, upper, lower, and periphery) of each animal were obtained to ensure thorough analysis because histologic confirmation of clinical OB lesions is often extremely difficult to detect. A semiquantitative grading system was used to assess severity of airway injury:

Grade 1-Bronchial epithelial denuding, mild to moderate inflammation within the airway wall and surrounding interstitium.

Grade 2-Bronchiolar epithelial denuding, destruction of the elastica and muscular wall hyperplasia with extensive inflammation extending into peribronchiolar tissue.

Grade 3-Intraluminal cellular proliferation, young granulation tissue and fibrosis with partial occlusion of the airway (less than $50 \%$ of lumen diameter).

Grade 4 -Intraluminal projection or plugging with young granulation tissue and fibrosis with obliteration of at least $50 \%$ of airway

Sections were graded blindly by four independent observers.

Statistical analysis. Grade of airway injury is presented as means \pm standard deviation. Differences between groups were assessed by analysis of variance, with Fisher's PLSD correction for multiple comparisons, with a commercial statistical software program (Slatview 4.0).

\section{Results}

\section{Part I}

Determining the optimal dose of activated charcoal. Four weeks after transtracheal installation of $0.1 \mathrm{ml}$ of activated charcoal, evidence of mild focal airway epithelial injury and inflammation was noted. Animals receiving $0.2 \mathrm{ml}$ of charcoal demonstrated mild to moderate inflammation of the terminal airways with associated submucosal inflammation. None of these animals died as a result of treatment within this dose range. Animals receiving $0.3 \mathrm{ml}$ or $0.4 \mathrm{ml}$ of activated charcoal demonstrated similar histologic changes as animals receiving $0.2 \mathrm{ml}$. However, 4 of the 10 animals in these groups combined died acutely after installation of charcoal. Therefore 
we chose $0.2 \mathrm{ml}$ of activated charcoal as the optimal dose for our animal model.

Pathologic evolution of airway injury

ACUTE Inflammatory PHASE ( 2 to 4 weEks). On low-to-medium magnification, charcoal particles were observed to be randomly deposited within the lumen and submucosa of bronchioles, respiratory bronchioles, and alveolar ducts. On higher magnification, charcoal-laden bronchioles demonstrated evidence of moderate acute nonspecific airway inflammation and injury. Admixture of inflammatory cells, including lymphocytes and polymorphonuclear cells, was observed histologically (Fig. 1, A). OB-like lesions were not observed at this time. No apparent injury was detected in charcoal-free bronchioles. Nonspecific airway, vascular, and interstitial edema was detected (Fig. 1, B) in lungs injected with sorbitol solution. Airway obliteration was not detected.

Proliferative phase (12 to 20 weeks). In charcoal-laden bronchioles focal epithelial sloughing and replacement by cuboidal-like cells were detected. In the submucosa the smooth muscle layer was often disrupted by proliferation of granulation tissue. Progressive intraluminal infiltration by inflammatory cells, fibroblasts, and fibrous tissue ensued, resulting in varying degrees of airway obliteration (Fig. 1, C). These obliterative airway lesions were observed focally in four of five charcoaltreated animals at 12 weeks and in all charcoaltreated animals by 16 and 20 weeks. Patchy areas of atelectasis and alveolar interstitial thickening were also observed in the parenchyma. Charcoal-free airways were essentially normal without compromise to the lumen.

At 16 to 20 weeks after sorbitol administration, animals demonstrated near normal recovery of alveolar septum, airways, and vasculature (Fig. 1, D). OB-like lesions were not observed in any of the sorbitol-treated animals.

Part II. In this experiment we assessed whether chronically rejecting rat lung allografts are more susceptible to airway injury and development of obliterative airway lesions when exposed to a nonallogeneic airway irritant (charcoal).

At 3 months after transplantation, chronically rejecting rat lung allografts (group I) demonstrated evidence of acute grade III to IV vascular rejection. Diffuse perivenular and periarterial cuffing by multiple layers of mononuclear cell infiltrates with extension into the adjacent alveolar septae was frequently observed. Pathologic abnormalities consistent with chronic vascular rejection were also observed as noted by endothelialitis, medial wall hyperplasia (Fig. 1,E), proliferation of the adventitial layer, and extensive neovascularization. In four of five allografts the distal respiratory airways demonstrated only mild reactive changes. This consisted of focal epithelial and peribronchial inflammation without evidence of OB lesions (Fig. 1, F). Late airway changes consistent with active $\mathrm{OB}$ were observed scarcely in only one of five allografts.

In charcoal-treated lung allografts (group II), charcoal particles were observed to be randomly deposited within the distal airways and admixed eccentrically with inflammatory cells, fibroblasts, and young granulation tissue. This resulted in development of diffuse obliterative airway lesions (Fig. 1, $G$ to $H)$. In the submucosa the smooth muscle layer was frequently disrupted by infiltrating inflammatory cells and fibrous tissue. These OB-like lesions were observed diffusely in all charcoal-treated allografts. Mean grade of airway injury (Fig. 2) in this group of animals $(3.7 \pm 0.36)$ was statistically significantly greater than group I $(1.63 \pm 0.35, p=$ $0.001)$ allografts. Evidence of acute and chronic vascular rejection was present with similar intensity and distribution as seen in group I.

In group III animals charcoal-laden bronchioles demonstrated epithelial sloughing over intraluminal charcoal particles focally admixed with infiltrating inflammatory cells and foreign body giant cells, resulting in focal airway obliteration. Mean grade of airway injury $(2.3 \pm 0.45)$ was statistically significantly $(p=0.04)$ less than charcoal-treated allografts (group II). The pulmonary arterial and venous system demonstrated mild inflammation with no compromise to lumen patency.

\section{Discussion}

Despite advances in early postoperative management of lung transplant recipients, a lack of basic understanding of the natural history and cause of OB still prevails.

In this study we initially sought to develop a reliable nonallogeneic animal model of OB-like lesions. On the basis of our histologic observations, the deposition of charcoal particles in the distal airways induced a process of ongoing subacute airway epithelial injury and submucosal inflammation. Infiltration by various inflammatory cell subsets to the charcoal-laden bronchioles likely stimulated the release of a variety of cytokines that can contribute to further injury and delay in regeneration. We are currently using immunohistochemical 

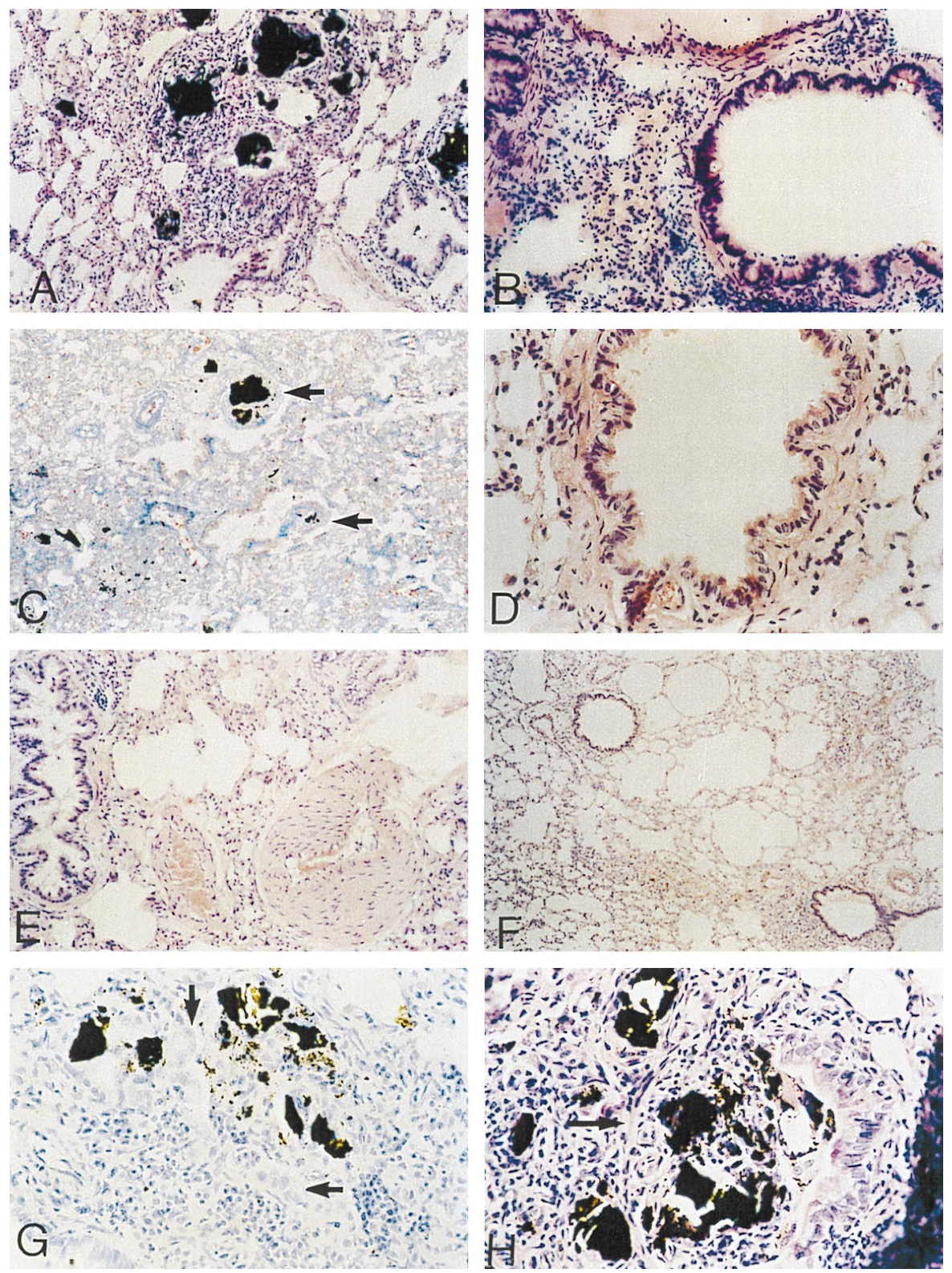

Fig. 1. Histologic observations in animal groups. Panel A (hematoxylin and eosin $\times 200$ ), Intraluminal and peribronchiolar charcoal particles with diffuse lymphocytic infiltrates and extension into adjacent alveolar septae during the acute inflammatory phase. Charcoal-free airways demonstrate no reactive changes. Panel B (hematoxylin and eosin $\times 250$ ), Diffuse peribronchiolar and interstitial edema with mild to moderate inflammatory cellular infiltrates at 2 weeks after sorbitol administration. Panel C (Masson's trichrome $\times 260$ ), Development of OB-like lesions in respiratory bronchioles (arrows) after charcoal treatment in native LEW lungs at 12 weeks. Panel D (hematoxylin and eosin $\times 400$ ), Normal recovery of lung parenchyma at 20 weeks after sorbitol instillation. Panel E (hematoxylin and eosin $\times 250$ ), Relatively unremarkable airway with severe medial wall hyperplasia of the vessels in chronically rejecting lung allografts $(3$ months). Panel $\mathbf{F}$ (hematoxylin and eosin $\times 100)$, Mild peribronchiolar and vascular reactive changes without evidence of OB in chronically rejecting rat lung allografts (3 months). Panel G (hematoxylin and eosin $\times 400$ ), OB-like lesions in charcoal-treated lung allografts (3 months). Note residual reactive bronchiolar epithelium (arrows). Panel $\mathbf{H}$ (hematoxylin and eosin $\times 450$ ), OB-like lesions with evidence of peribronchiolar smooth muscle disruption (arrow) in charcoal-treated lung allografts (3 months). 


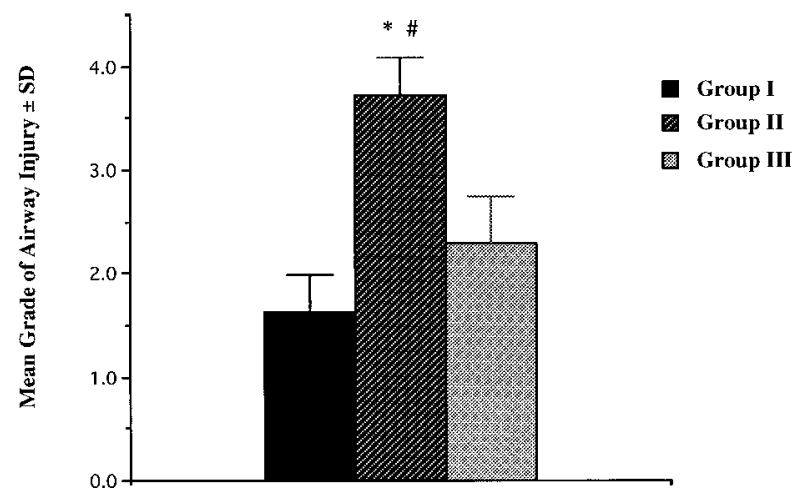

Fig. 2. Mean ( \pm SD). Semiquantitative grade of airway injury. Mean grade of airway injury was significantly greater in charcoal-treated allografts (group II) compared with both chronically rejecting lung allografts (group I) or charcoal-treated native lungs (group III). ${ }^{*} p=0.001$ vs group I, \#p $=0.04$ vs group III.

techniques to evaluate the contribution of several inflammatory cytokines. Preliminary unpublished data reveal strong diffuse immunostaining for cytokines IL- $1 \beta$, IL-8, and TNF- $\alpha$ up to 12 weeks after charcoal administration. Progressive transition from an acute inflammatory phase to a fibroproliferative one was observed at approximately 3 months. The persistence of charcoal particles in the airway lumen and submucosa augmented fibroblast infiltration and fibrosis, which ultimately led to obliteration of the distal airways. Animals receiving treatment with sorbitol solution also had early nonspecific inflammation of the lung with mild to moderate acute cellular infiltration develop. However, animals were able to demonstrate near normal recovery and resolution from the sorbitol-induced lesions. This suggests that the presence of activated charcoal is essential in stimulating chronic subclinical airway injury and inflammation around the airway.

The use of activated charcoal as a foreign airway irritant and marker has several advantages. First, it has the ability to target and induce ongoing nonspecific airway inflammation with reproducible development of obliterative airway lesions. Second, administering activated charcoal, as a nonallogeneic cofactor in experimental studies, is technically simpler and safer to use than infectious agents. Third, it is possible to quantitate the variety of cells, biochemical products, and cytokines that can collect in the bronchopulmonary compartment using bronchoalveolar lavage techniques. ${ }^{7}$ This feature may allow investigation into the various mechanisms involved in the development of OB. Finally, pulmonary physiologic measurements can be performed in these animal models. To this effect, we have begun preliminary pulmonary function tests in normal and charcoal-treated rats. Pulmonary function parameters analyzed include lung compliance, resistance, total lung capacity, and functional residual capacity.

After lung transplantation, host pulmonary defense and mucociliary clearance mechanisms are impaired. ${ }^{8}$ In this setting, heightened and prolonged exposure to harmful antigenic, infectious, or noxious agents can occur in the airway compartment. ${ }^{9-12}$ These particles may directly or indirectly stimulate secondary airway injury. Alternatively, these nonallogeneic stimuli may act as cofactors amplifying the damaging effects of chronic rejection. For this we demonstrated that instillation of activated charcoal into inadequately immunosuppressed rat lung allografts (group II) aggravates and induces progressive damage to the distal respiratory airways. We detected moderate to severe airway injury and inflammation with evidence of diffuse OB-like lesions in charcoal-laden allografted bronchioles. Severity and frequency of obliterative airway lesions was statistically significantly more pronounced in charcoaltreated allografts than in charcoal-treated native lungs (group III). It can be postulated that intact host defense and mucociliary clearance mechanisms in native lungs contributed to enhanced clearance of foreign and desquamated inflammatory products away from the distal airways. In contrast, without the addition of a nonspecific airway irritant, inadequately immunosuppressed rat lung allografts (group I) primarily had severe vascular changes develop consistent with acute and chronic vascular rejection. Most airways demonstrated only mild reactive changes. On the other hand, several investigators reported reproduction of $\mathrm{OB}$ lesions as a result of chronic rejection alone. ${ }^{13,14}$ These disparities may be explained by differences in the cause of $\mathrm{OB}$ in various animal strains and humans. With a porcine model of lung allotransplantation, Al-Dossari and investigators ${ }^{13}$ demonstrated that tapering and discontinuation of immunosuppression results in progressive fibrous occlusion of bronchioles consistent with OB-like lesions. Immunohistochemical staining demonstrated increased expression of major histocompatibility complex class II antigen off the bronchiolar epithelium and increased dendritic cells and CD4+ lymphocytes around affected airways. In a mouse model of heterotopic tracheal transplantation, Hertz and colleagues ${ }^{14}$ observed 
fibroproliferation of the airway walls in most allografts. Taking our findings together with these reports, we suggest that the pathogenesis of posttransplant OB is likely to be caused by nonallogeneic stimuli, allogeneic stimuli, or both factors.

In conclusion, this study describes a novel animal model of charcoal-induced airway inflammation, stimulating development of OB-like lesions in native and transplanted lungs. The histologic features of the obliterative airway lesions and course of the disease process is reproducible and resembles in many ways what is observed in other experimental models of OB. This animal model may provide a method to independently study the mechanisms involved in the pathogenesis of OB in a nonallogeneic setting. Alternatively, this model may also be used to investigate the mechanisms associated with the development of OB caused by allogeneic and nonallogeneic stimuli. Finally, we demonstrated that subjecting chronically rejecting rat lung allografts to secondary nonspecific airway inflammation results in severe airway injury and diffuse development of obliterative airway lesions. The effects of chronic rejection alone were not sufficient to induce permanent airway injury because OB lesions were almost nonexistent. This finding suggests that nonspecific airway inflammation, induced by secondary nonallogeneic stimuli, may be necessary for the development of diffuse OB lesions after lung transplantation.

\section{REFERENCES}

1. Reichenspurner H, Girgis RE, Robbins RC, et al. Obliterative bronchiolitis after lung and heart lung transplantation. Ann Thorac Surg 1995;60:1845-53.

2. Milne DS, Gascoigne AD, Ashcroft T, et al. Organizing pneumonia following pulmonary transplantation and the development of obliterative bronchiolitis. Transplantation 1994;57:1757-62.

3. Reid KR, McKenzie FN, Menkis AH, et al. Importance of chronic aspiration in recipients of heart-lung transplants. Lancet 1990;336:206-08.

4. Tullius SG, Heemann UW, Azuma A, et al. Antigen-independent events mimic characteristic changes of chronic allograft rejection. Transplant Proc 1995;27:561-3.

5. Stewart S. Critical summaries in lung transplant pathology. J Pathol 1994;173:135-43.

6. Reis A, Giaid A, Serrick C, Shennib H. Improved outcome of rat lung transplantation with modification of the nonsuture external cuff technique. J Heart Lung Transplant 1995;14: 274-9.

7. Reis A, Totsch M, Shennib H, et al. Bronchoalveolar lavage: results of sequential, selective techniques in viable murines. Am J Respir Crit Care Med 1994;150:547-50.

8. Shennib H. Chronic irreversible graft dysfunction (CIGD) and animal models of obliterative bronchiolitis. In: Shennib $\mathrm{H}$, editor. Medical intelligence unit: immunology of the lung allograft. Austin (TX): RG Landes Company; 1995. p. 12130.

9. Churg AM. Mineral dust induced bronchiolitis. In: Epler GR, editor. Diseases of the bronchioles. New York: Raven Press, 1994. p. 139-52.

10. Elliot CG, Colby TV, Kelly TM, Hicks HG. Charcoal lung: bronchiolitis obliterans after aspiration of activated charcoal. Chest 1989;96:672-4.

11. Kirk AJ, Colquhoun IW, Cords PA, et al. Impaired gastrointestinal motility in pulmonary transplantation. Lancet 1990; 336:752.

12. Rinaldi M, Martinelli L, Volpato G, et al. Gastro-esophageal reflux as cause of obliterative bronchiolitis: a case report. Transplant Proc 1995;27:2006-7.

13. Al-Dossari GA, Kshettery VR, Jessurun J, Bolman RM III. Experimental large-animal model of obliterative bronchiolitis after lung transplantation. Ann Thorac Surg 1994;58:34-9.

14. Hertz MI, Jessurun J, King MB, et al. Reproduction of the obliterative bronchiolitis lesion after heterotopic transplantation of mouse airways. Am J Pathol 1993;142:1945-51. 